\title{
Efficient synthesis of biobased glycerol levulinate ketal and its application for rigid polyurethane foam production
}

\section{Supporting Information}

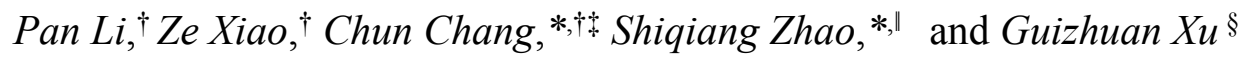

†School of Chemical Engineering and Energy, Zhengzhou University, Science Road 100, Zhengzhou 450001, China;

Key Laboratory of Biomass Chemical Engineering of Ministry of Education, Zhejiang University, Hangzhou 310027, China;

"Department of Chemical and Biochemical Engineering, College of Chemistry and Chemical Engineering, Xiamen University, Xiamen, 361005, China;

${ }^{\S}$ College of Mechanical and Electrical Engineering, Henan Agricultural University, Zhengzhou, 450002, China. 
Table S1. GLK Optimization Experimental Design and Results

\begin{tabular}{ccccc}
\hline No & Molar ratio $\left(X_{1}\right)$ & Temperature $/{ }^{\circ} \mathrm{C}\left(X_{2}\right)$ & $\begin{array}{c}\text { Vacuum degree / } \\
\mathrm{kPa}\left(X_{3}\right)\end{array}$ & $\begin{array}{c}\text { GLK yield } / \% \\
(Y)\end{array}$ \\
\hline 1 & 4 & 120 & 90 & 96.7 \\
2 & 3 & 110 & 90 & 83.9 \\
3 & 4 & 110 & 92 & 90.4 \\
4 & 5 & 120 & 88 & 94.1 \\
5 & 4 & 110 & 88 & 86.4 \\
6 & 4 & 120 & 90 & 96.9 \\
7 & 5 & 110 & 90 & 91.1 \\
8 & 5 & 120 & 92 & 94.0 \\
9 & 3 & 130 & 90 & 90.8 \\
10 & 4 & 120 & 90 & 96.4 \\
11 & 5 & 130 & 90 & 94.8 \\
12 & 3 & 120 & 92 & 90.6 \\
13 & 4 & 130 & 92 & 94.1 \\
14 & 4 & 120 & 90 & 96.1 \\
15 & 3 & 120 & 88 & 87.4 \\
16 & 4 & 120 & 90 & 96.5 \\
17 & 4 & 130 & 88 & 92.7 \\
\hline
\end{tabular}


Table S2. ANOVA for the Regression Model

\begin{tabular}{llllll}
\hline Source & $\begin{array}{l}\text { Sum of } \\
\text { square }\end{array}$ & $\begin{array}{l}\text { Degree of } \\
\text { freedom }\end{array}$ & Mean squares & $F$ value & $p$ value \\
\hline Model & 241.32 & 9 & 26.81 & 150.11 & $<0.0001$ \\
$X_{1}$ & 7.23 & 1 & 7.23 & 40.46 & 0.0004 \\
$X_{2}$ & 5.44 & 1 & 5.44 & 30.45 & 0.0009 \\
$X_{3}$ & 20.77 & 1 & 20.77 & 116.26 & $<0.0001$ \\
$X_{1} X_{2}$ & 2.56 & 1 & 2.56 & 14.33 & 0.0068 \\
$X_{1} X_{3}$ & 2.76 & 1 & 2.76 & 15.43 & 0.0057 \\
$X_{2} X_{3}$ & 1.76 & 1 & 1.76 & 9.83 & 0.0165 \\
$X_{1}^{2}$ & 34.99 & 1 & 34.99 & 195.89 & $<0.0001$ \\
$X_{2}^{2}$ & 50.85 & 1 & 50.85 & 284.69 & $<0.0001$ \\
$X_{3}^{2}$ & 18.93 & 1 & 18.93 & 105.97 & $<0.0001$ \\
Residual & 1.25 & 7 & 0.18 & & \\
Lack of Fit & 0.88 & 3 & 0.29 & 3.19 & 0.1458 \\
Pure error & 0.37 & 4 & 0.092 & & \\
Cor Total & 242.57 & 16 & & & \\
$R$-squared & 0.9948 & & & & \\
CV\% & 0.46 & & & & \\
\hline
\end{tabular}


Table S3. Formulation of PU Foams. All Formulation Are Based on 100 Parts by Weight of Polyol (php)

\begin{tabular}{lllll}
\hline Component & $\mathrm{PU}_{0}$ & $\mathrm{PU}_{75}$ & $\mathrm{PU}_{100}$ & $\mathrm{PU}_{100} / \mathrm{D}$ \\
\hline Polyol 4110 & 100 & 25 & 0 & 0 \\
Biopolyol & 0 & 75 & 100 & 100 \\
Polycat $^{\circledR} 5$ & 1.11 & 1.11 & 1.11 & 1.11 \\
Polycat $^{\circledR 8} 8$ & 0.75 & 0.75 & 0.75 & 0.75 \\
$\mathrm{AK8805}$ & 2.16 & 2.16 & 2.16 & 2.16 \\
Deionized water & 2.5 & 2.5 & 2.5 & 2.5 \\
Diatomite & 0 & 0 & 0 & 3 \\
pMDI & \multicolumn{5}{r}{} \\
\hline
\end{tabular}




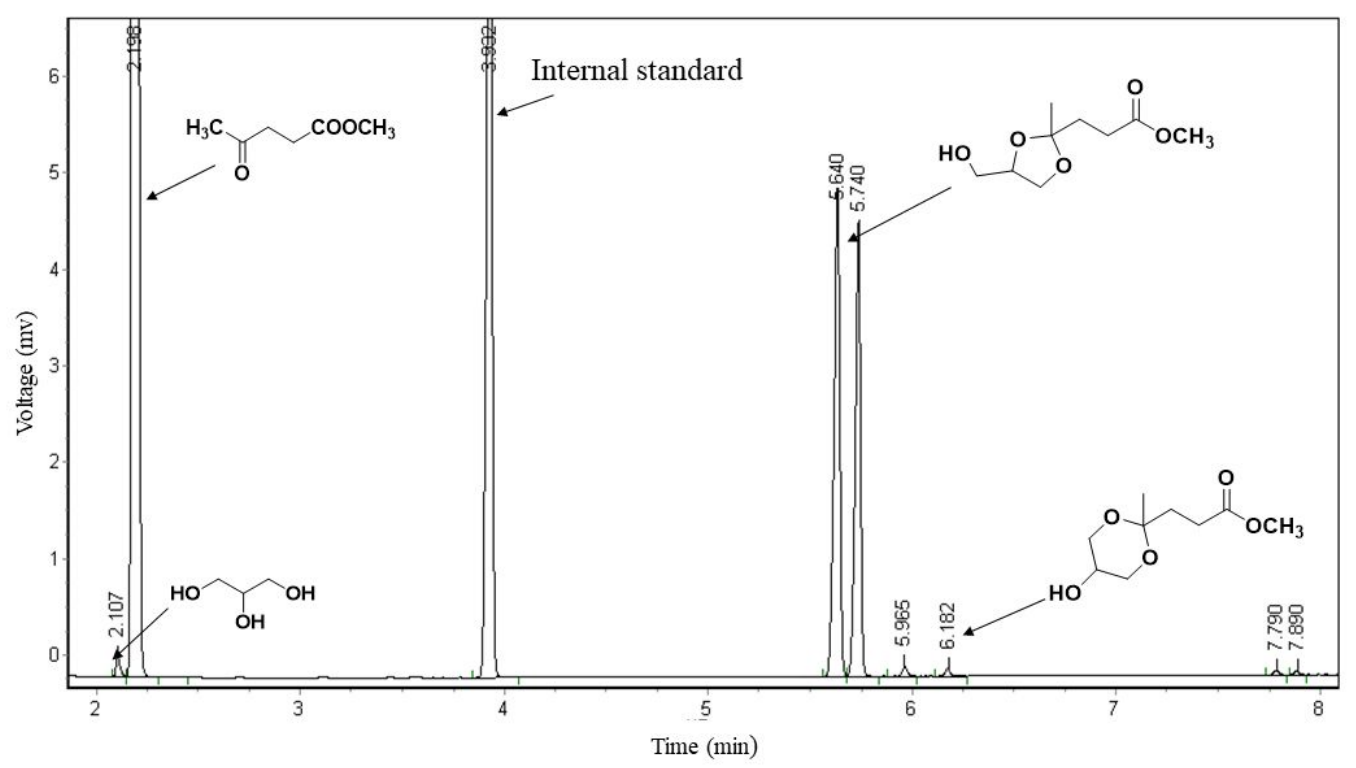

Figure S1-1. GC chromatograms of the ketalization reaction mixture

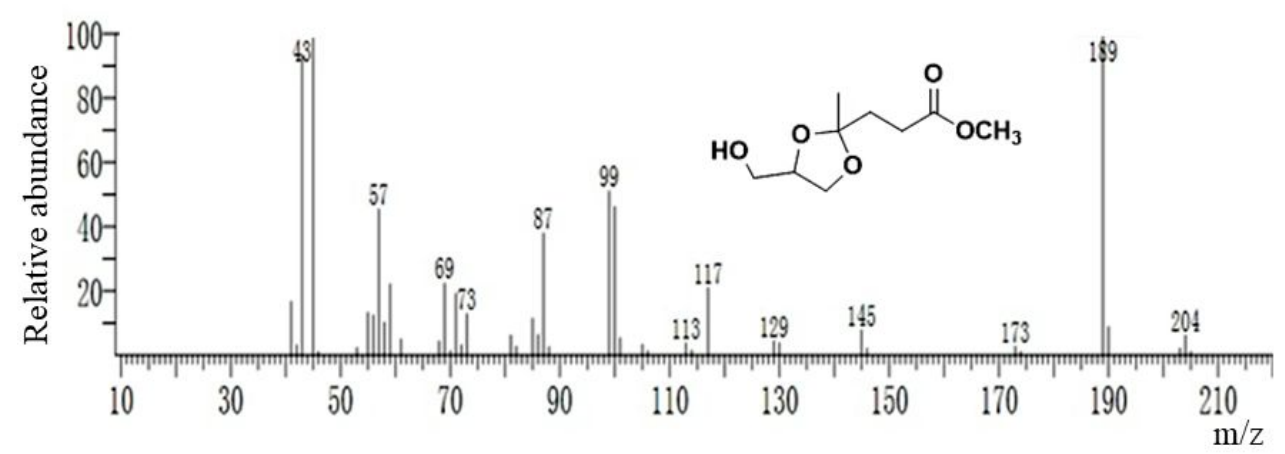

Figure S1-2. MS of GLK 


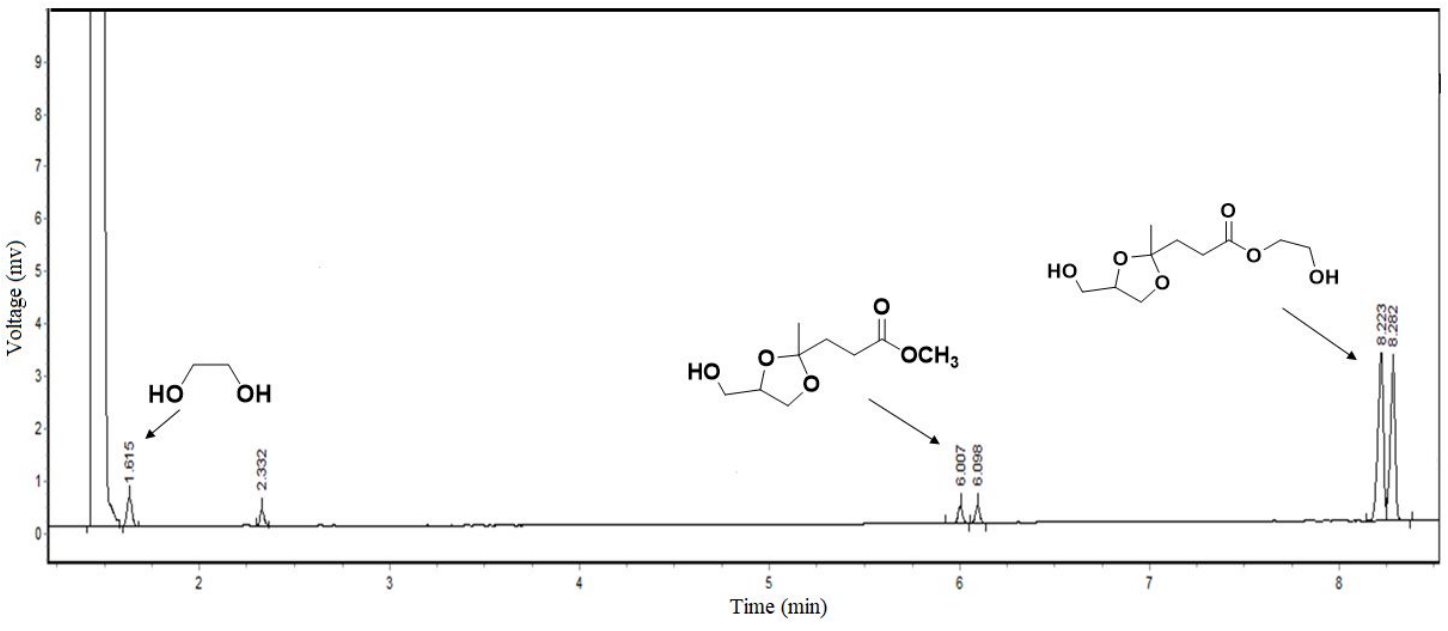

Figure S2. GC chromatograms of the transesterification reaction mixture 